\title{
Commentary: Remote electronic consultation for COVID-19 patients in teaching hospitals in Tehran, Iran
}

\author{
Seyed Hamid Reza Faiz ${ }^{1}$, Taghi Riahi ${ }^{1}$, Poupak Rahimzadeh², Nasim Nikoubakht*1 (1) \\ Received: 24 Mar 2020 \\ Published: 8 Apr 2020 \\ Conflicts of Interest: None declared \\ Funding: None \\ *This work has been published under CC BY-NC-SA 1.0 license. \\ Copyright $₫$ Iran University of Medical Sciences
}

Cite this article as: Faiz SHR, Riahi T, Rahimzadeh P, Nikoubakht N. A solution: Commentary: Remote electronic consultation for COVID-19 patients in an academic hospital. Med J Islam Repub Iran. 2020 (8 Apr);34:31. https://doi.org/10.47176/mjiri.34.31

In late 2019, a cluster of pneumonia cases caused by a new coronavirus from Wuhan, China, has spread globally. Studies show that this disease has initially spread through the hospitals or medical centers contaminated with the virus. Doctors, nurses, and other health care providers may be at the particular risk of this outbreak. Job immunity of the health care providers is one of the key principles of health care ethics. In this regard, protocols have been developed to reduce this risk in hospitals or medical centers to limit the chain of infection transmission in the health care provider and the family. Using appropriate personal protective equipment (PPE) such as gloves, protective clothing, masks, face shields, or eye protection for doctors, nurses, and other health care providers are highly recommended. All objects in the coronavirus wards such as files, pens, desks, chairs, and other items in patients' rooms are contaminated with the virus. Thus, for less exposure, hospitals must undertake clinical services management (CSM) activities, such as teleworking if possible, leading to a reduction in staff attendance in coronavirus wards (1-3). Iran University of Medical Sciences and Health Servives coveres a population of approximately 5 million people, including 4.700 million in urban areas and 300000 in rural areas with several teaching and nonteaching hospitals. The main teaching hospital (Rasoul Akram hospital) covers many catchment areas in west and center of capital of Iran with different hospitals. These smaller hospitals lack skilled specialists needed in the patient management, and this is while we should both protect the health of our skilled specialists and help others. Accordingly, the aim of this study was to reduce the risk of COVID-19 exposure in doctors and medical professionals who attended the coronavirus wards to provide consultations.

A variety of technologies, including telemedicine, online web-based methods, and electronic medical records have been used for remote consultation. Doctors can treat COVID-19 patients without visiting them (4). Keely et al (2013) found that the use of e-consultation system in Canadian hospital network reduced both the presence of doctors and the wasted time for the patients, which was performed using a web-based e-consultation system (5) .In Hannah B Edwards study (2019), e-consultation system was investigated in the United Kingdom's hospital network and the results showed a reduction in staff workload and a decrease in the waiting time (6) .In this regard, authorities at Rasoul Akram hospital decided to develop an electronic health records system and remote consultation for the COVID-19 patients. We prepared this access in Hospital Information System (HIS). For the first time, this system was developed during the outbreak of disease and its main purpose is to limit the attendance of doctors and medical professionals in coronavirus wards. Our decision on developing the system was based on the opinions obtained from a team consisting of faculty members and experts after examining different methods. Advantages of this system are as follow: (1) to control the exposure rate for specialist physicians by limiting their attendance at coronavirus wards; (2) to eliminate the volume of the patients' medical files, which in turn reduces costs; and (3) to provide a faster consultation services for the patients; and (4) to reduce wasting personal protective equipment. However, the disadvantages of this system were as follow: (1) Many physicians did not tend to use this system and preferred face-to-face examination, and (2) lack of enough knowledge to access the telemedicine system and work

\footnotetext{
Corresponding author:Dr Nasim Nikoubakht, nikoobakht.n@iums.ac.ir

1. Rasoul Akram Hospital Clinical Research Development Center (RCRDC), Rasoul Akram Hospital, Iran University of Medical Sciences, Tehran, Iran

2. Pain Research Center, Rasoul Akram Hospital, Iran University of Medical Sciences, Tehran, Iran
} 
with it. Thus, efforts should be done to train physicians and convince them that this system is more effective and safer for the existing situation (7). The Deputy of Education at Rasoul Akram hospital network provided training for the use of this system to all the physicians and learners via online training video clips launched and released through hospital networks (3). This system is difficult to access if there are technical difficulties; therefore, software companies have been informed of such problems to solve them.

Based on the needs assessment, we used e-consultation and telemedicine system to evaluate the area of need and designed the following programs:

\section{(1) An office-based program}

Using this system, we obtained the necessary consultations from senior skilled physicians who had underlying medical conditions and thus were at a higher risk for serious complications from COVID-19. They provided us with their expertise and experience, while they stayed at their own office.

\section{(2) A home-based program}

Using telemedicine with a team consisting respiratory, infectious disease, and radiologist specialists helps the emergency medicine physicians to provide better care to patients. We tried to achieve an accurate and rapid triage of patients. In case of a need for specialized home-based interventions, we provided necessary guides and prescribed treatment online. Using this system, we can support smaller hospitals that lack highly skilled specialists; moreover, patients are less likely to be hospitalized.

\section{(3) A hospital-based program}

An e-consultation system was developed in the Rasoul Akram hospital. This system includes various forms, including patients' medical history, progress notes forms, a consultation sheet, all diagnostic tests, and procedures.

The consulting physician can have access to all this information using computers in each ward, physicians' room, and even library and their pavilion using their own password and they can observe all patients' information, including a history of symptoms, the course of illness, and patient radiographs, and tests. A consultation file is presented in each file folder and can be observed after it is opened. The specialist gives orders after studying the file. The responsible physician working in the coronavirus ward will implement the instructions given in the electronic consultation form.

\section{(4) Patient Follow-up}

Many patients still need help after being discharged from the hospital. Thus, using telemedicine, a team of specialists consisting of highly skilled psychiatrists and trained medical assistant interns will assist them. They will also evaluate the patients after being discharged from the hospital and obtain information about their physical and psychological health status and their family at home.

One problem of this program was related to the health insurance payment system, because consultation sheets must be signed and sealed by a specialist physician, which is also a problem in the electronic consultation system. However, this problem was solved after making arrangements with the health insurance companies to accept electronic signature for the coronavirus wards.

\section{Conclusion}

Designing remote consultation services can reduce the presence of specialist physicians and physician assistants in coronavirus wards, which in turn reduces the risk of infection for the hospital staff, decreases the waiting time, and reduces the number of personal protective equipment.

\section{Acknowledgment}

The authors wish to thank Rasoul Akram Hospital Clinical Research Development Center (RCRDC), Iran University of Medical Sciences for its editorial assistance.

\section{Conflict of Interests}

The authors declare that they have no competing interests.

\section{References}

1. Bly RA, Bly EG. Consult Courtesy. J Grad Med Educ. 2013;5(3):5334.

2. Mclnosh K. Coronavirus disease 2019 (COVID-19). UpToDate. Last Updated. 2020;09.

3. Hong YR, Lawrence J, Williams D Jr, Mainous Iii A. PopulationLevel Interest and Telehealth Capacity of US Hospitals in Response to COVID-19: Cross-Sectional Analysis of Google Search and National Hospital Survey Data. JMIR Public Health Surveill. 2020;6(2):e18961.

4. Dehghani M, Nooshiravani Y, Shokrizadeh-Bezenjani K, Asgarian A. The Problems of Health Information Management Departments and their Causes in Iran. Health Inf Manag. 2019;15(6):253-9.

5- Keely E, Liddy C, Afkham A. Utilization, benefits, and impact of an e-consultation service across diverse specialties and primary care providers. Telemed J E Health. 2013;19(10):733-8.

6. Edwards HB, Marques E, Hollingworth W, Horwood J, Farr M, Bernard $\mathrm{E}$, et al. Use of a primary care online consultation system, by whom, when and why: evaluation of a pilot observational study in 36 general practices in South West England. BMJ Open. 2017;7(11):e016901.

7. Portnoy J, Waller M, Elliott T. Telemedicine in the Era of COVID-19. J Allergy Clin Immunol Pract. 2020. 